\title{
Non-uniform Cluster-Based Mobile Data Collector Routing Protocol in Wireless Sensor Networks
}

\author{
Xinlian Zhou \\ School of Computer Science and Engineering, Hunan \\ University of Science and Technology University \\ Xiangtan, china \\ E-mail: zhouxinlian68@sina.com
}

\author{
Sheng Chen \\ School of Computer Science and Engineering, Hunan \\ University of Science and Technology University \\ Xiangtan, china \\ E-mail: cshengcs@sina.cn
}

\begin{abstract}
One fundamental task of wireless sensor networks (WSNs) is to save sensor power, and try to prolong the lifetime of WSNs. Traditional routing protocols are almost based on homogeneous WSNs, all sensors are use single hop or multi-hop to transmit data to base station, which lead to overload sensors next to base station, thus appear energy hole and made WSN outwork. In this paper, we use a new novel mobile collector's mobile strategy method to prolong network lifetime, and reduce hot-spot effect around base station. We propose the routing method can effectively balance the network sensors energy depletion, more save energy and longer whole network lifetime compare to LEACH.
\end{abstract}

Keywords-wireless sensor network, mobile data collector, routing protocol.

\section{Introduction}

In traditional wireless sensor deployments, nodes usually depend on single hop and multi-hop forwarding mechanism to complete the data collection. When a sensor node is far away from the base station, the data will be forwarded to the neighboring nodes of the base station [1]. However, at this time, nodes close to the base station have to take on more data forwarding work and prone to the energy hole, shortening the life cycle of the entire network.

To solve this problem, we can use the method of introduction of mobile elements [2], we can also use the network node clustering method. Mobile element move in node deployment region and forward the data from node to base station $[3,4]$. In this paper, we combine the clustering and mobile elements (mobile collector) methods to ease the nodes workload within the hot zone, which near the base station can early death due to excessive energy consumption, thus extending the life cycle of the network.

\section{Network Model}

Network assumed as follows:

1) The radius of all the sensor nodes and base station are located within a circular area of $\mathrm{R}$, the base station located in the center of the circle and remain stationary, and once after the deployment.

2) All sensor nodes are evenly distributed, and relatively independent of each node has a duplicate identifier (ID).
3) The sensor nodes based on whether their monitoring event occurred or not, in the two states. If sensitive event does not occur then in the sleeping state, otherwise active.

4) The maximum transmission distance of every sensor node is $d_{0}$, and is also the upper limit. It can be free to adjust the transmission distance.

5) Mobile collector has the rechargeable energy features and equipped with the GPS function with self-positioning move.

\section{Network clustering process and analysis}

A. Clustering of sensor nodes in the network

Clustering method provides an effective way to extend the life cycle of wireless sensor networks. Traditional clustering protocol, such as LEACH, it use of two techniques: the random election of cluster head and the periodic replacement of the cluster head. Consider of non-clusters, the network node generated data to be transmitted directly to the base station shown in Figure 1. In the sub-cluster case, the nodes in the network will send data to the cluster head node belongs, and then the cluster head collecting data integration to send to the base station shown in Figure 2.

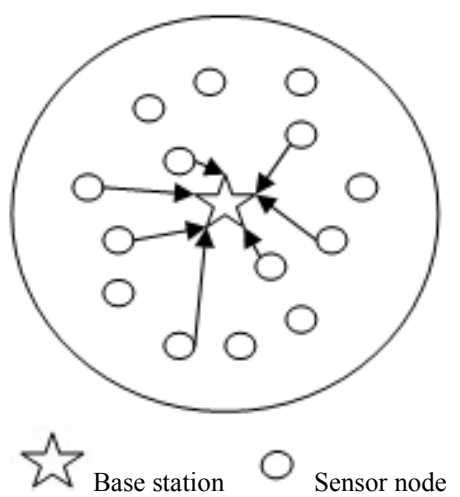

Figure 1. Wireless Sensor Network without cluster, sensor nodes send data direct to basestation. 

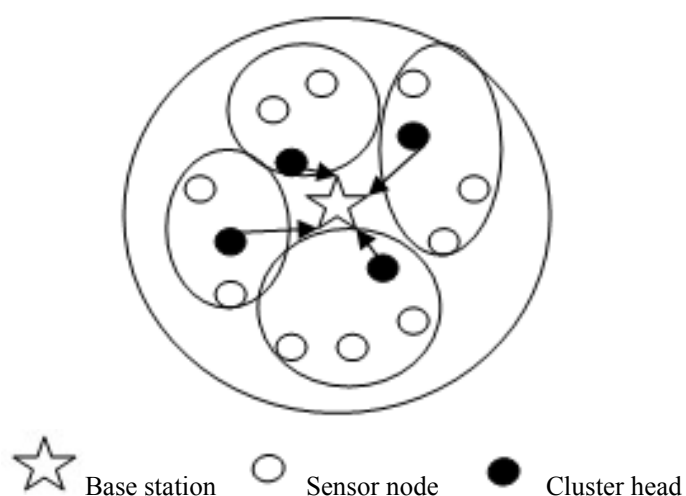

Figure 2. Wireless Sensor Network with cluster, sensor nodes send data to cluster heads, and then cluster heads send data to basestation.

This article is also based on clustering methods, known as LEACH. In the LEACH algorithm cluster head election phase, the node randomly generates a value between 0 and 1, and compare with the network of the current round threshold if less than it then be the cluster head. $T(n)$ is calculated as follows [5]:

$T(n)=\left\{\begin{array}{lc}\frac{P}{1-P^{*}\left(r \bmod \frac{1}{P}\right)} & \text { if } n \in G \\ 0 & \text { other }\end{array}\right.$

Where $P$ is the ratio of the cluster head node, $r$ is the current round and $G$ is not yet act as a cluster head node collection in the remaining $\frac{1}{P}$ round. Use this threshold to ensure that each node can act as a cluster head in $\frac{1}{P}$ round. In this case, each node becomes the cluster head probability is the same, so this method of clustering is uniform clustering method. Then we have taken a non-uniform clustering.

\section{B Non-uniform clustering process}

Uniform clustering conditions compared to the cluster head away from the base station deplete their energy more easily. Inspired, we asked the uneven clustering, so that the number of cluster head located near a base station is more than the number of cluster head away from the base station, thus consider the distance factor. We have changed the original LEACH cluster head election mechanism, the node to be elected as cluster head node when it randomly generated value bigger than the threshold value, in the contrary, not elected. Nodes generate random values are between 0 and 1 , then the threshold formula $T(n)$ as following shown:

$$
T(n)=\left\{\begin{array}{cl}
\frac{\frac{1}{P}-\left(\mathrm{r} \bmod \frac{1}{P}\right)-1}{\frac{1}{P}} & \text { if } n \in G \\
1 & \text { other }
\end{array}\right.
$$

Parameters similar to LEACH, only threshold $T(n)$ will decrease with the increase of the number of rounds $r$, then increases the node which not elected as cluster head to be become the cluster head 's probability, just the opposite with the LEACH clustering mechanisms. Next, more specific description of non-uniform clustering process.

The distance of the farthest node from the base station in the circular area marked as $d_{\text {max }}$, the distance nearest from the base station node labeled $d_{\text {min }}$, all nodes within a circular area named the set $\mathrm{S}$, one node, such as i-nodes labeled $S_{i}$, apparently $S_{i} \in S$. The distance between Node $S_{i}$ and the base station is labeled $d_{\left(S_{i}-B S\right)}$, then we based on the idea that add nodes at the base station near the cluster head number, and put forward a distance factor cluster head election mechanism, lead the node near by the base station to become cluster head's probability higher than the node far away from the base station, which will increase the number of nodes near the base station to be cluster head, then the uneven clustering threshold formula is as follows:

$$
T(n)= \begin{cases}\frac{\frac{1}{P}-\left(\mathrm{r} \mathrm{mod} \frac{1}{P}\right)-1}{\frac{1}{P}} *\left(1-c * \frac{d_{\max }-d_{\left(S_{i}-B S\right)}}{d_{\max }-d_{\min }}\right) & \text { if } n \in G \\ 1 & \text { other }\end{cases}
$$

Which $c$ is the regulator and its value between 0 and 1 , can regulate the ratio of cluster head number which nearly to the base station and remote to base stations. From this formula we can be seen, when a node within the network such as the node $S_{i}$ from the base station closer, then the value of $d_{\left(S_{i}-B S\right)}$ is smaller, In particular, if $S_{i}$ is exactly equal to the nearest node of the network from the base station, then $d_{\left(S_{i}-B S\right)}=d_{\text {min }}$, thereby $c * \frac{d_{\max }-d_{\left(S_{i}-B S\right)}}{d_{\max }-d_{\min }}=c$, take the higher value of $c$, then 1- $c$ is smaller, so $T(n)$ is also smaller, then higher the probability of the node to become cluster head. Conversely, if the node $S_{i}$ is the furthest from the base station, then $d_{\left(S_{i}-B S\right)}=d_{\max }$, thereby $c * \frac{d_{\max }-d_{\left(S_{i}-B S\right)}}{d_{\max }-d_{\min }}$ $=0$, the distance factor has no impact on the threshold value. We can draw from the above analysis that the closer the 
node to the base station, the greater the $c * \frac{d_{\max }-d_{\left(S_{i}-B S\right)}}{d_{\max }-d_{\min }}$ value is, and then the value of $1-c * \frac{d_{\max }-d_{\left(S_{i}-B S\right)}}{d_{\max }-d_{\min }}$ is smaller, thus the smaller the threshold $T(n)$ is, then the node randomly generated value between 0 and 1, higher than the threshold's probability is greater, thus ensuring the higher probability of node which closer to the base station to become the cluster head node, and the smaller the farther away from the base station.

IV. Non-uniform cluster-based Mobile data collector routing protocol

Previously proposed clustering protocol are based isomorphism sensor networks, this paper introduces a new heterogeneous nodes, called a mobile data collector. It equipped with GPS and has enough energy to support the function to be able to free movement. The introduction of the mobile data collector aims to ease the hot zone near the base station, and avoid the region of the energy hole that lead to network failure.

Transmission energy consumption of nodes in the network using the first wireless model, where node through the amplifier before the energy consumed with the transmission units of the packet is the same as that to accept the energy consumed by the unit packets, is assumed to $E_{\text {elec }}$, then the sender needs to send data out, and is bound to the amplifier with the signal amplification, their energy consumption using the following formula:

$$
E_{T x}(k, d)= \begin{cases}k^{*} E_{\text {elec }}+k^{*} E_{f s} * d^{2} & d<d_{0} \\ k^{*} E_{\text {elec }}+k^{*} E_{m p} * d^{4} & d \geq d_{0}\end{cases}
$$

Among them, $E_{\text {elec }}$ stand for the energy consumption which node sending unit data packet, $E_{f_{s}} 、 E_{m p}$ is the amplification factor of the amplifier. When the inter-node transmission distance $d<d_{0}$, use a free transfer model, on the contrary, the use of multi-path attenuation model. When node receives the $\mathrm{K}$-unit data packet, for example, it receives energy consumption formula is as follows:

$$
E_{R x}(k)=k^{*} E_{\text {elec }}
$$

Cluster head that far away from the base station collects the information within the cluster after data aggregation. It could adopt any one of the two transmission ways, and the multi-path attenuation model of energy consumption is much higher than the free-transfer model. Cluster head selected lower energy consumption of the free transmission in data transmission through multi-hop transmission way, then send data to cluster head nearby the base station, and the cluster head near the base station finished data integration, then sent data to the base station. Therefore, close to the base station cluster head needs to take on more data forwarding tasks, compared to the cluster head energy consumption away from the base station used quickly this time. For this reason, we have introduced a mobile collector which is responsible for monitoring the base station. Near the cluster head establishment phase and data transmission phase will move the mobile device to the cluster head with a relatively small energy in each round. As Figure 3 shows.
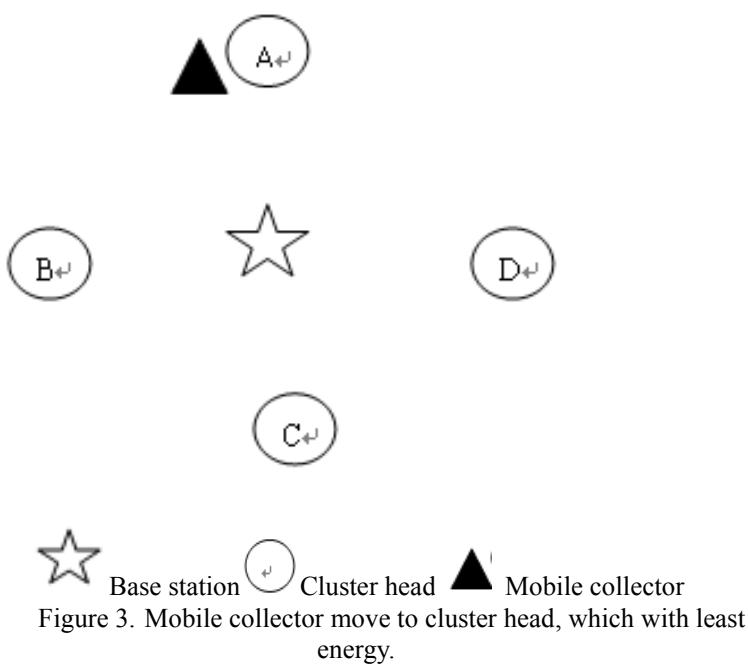

When the sensor network runs after a certain number of rounds, near the base stations cluster head that residual energy disparities. For example, if we consider A, B, C, D are cluster head, and located near a base station, and the A cluster head energy is minimum. Then the mobile collector is moved to the vicinity of the A cluster head, and take on the task of the cluster head A. This process is described as follows:

1) First, determine the area close to the base station. Assume that the entire circular area of radius is $\mathrm{R}$; the near-station factor named $\alpha$, it value is between 0 and 1 , through the base station to adjust it. Then established near the station's area radius is $R_{0}=\alpha^{*} R$.

2) Next, is the cluster head election process, after the cluster head establishment phase, cluster head sending the established information to the base station. Once the base station receives the information then broadcast cluster head request information with radius $R_{0}$, and once cluster head receives the information, then sends the remaining energy and the ID to their base station.

3) After the base station receives information from the cluster head, found the lowest energy cluster head corresponding to the ID by comparing, and then sends 
this ID to the mobile collector, once mobile collector receives the ID, by using the GPS positioning function, help mobile collector move to the ID corresponding to the cluster head's adjacent.

Due to unequal cluster head of energy near the base station, with the start of each round, the moving position of the mobile collector are not the same. With time goes, the path of the mobile collector approximated around the concentricity of the base station likes circular trajectory as show in Figure 3.

Therefore, those cluster head that far from the base station using a multi-hop than the single hop data transmission to the base station with more energy efficient. After several rounds, these cluster head energy inequality nearby the base station, the mobile collector receives the ID number of the cluster head and compare with residual energy, brush to select a relatively small energy cluster head, and then rely on GPS functionality to move around it. Since at the beginning of each round the location of the mobile collection are not the same, but in general, with the passage of time, the mobile collector moves around the base station path will be similar to the concentric track as Figure 4.

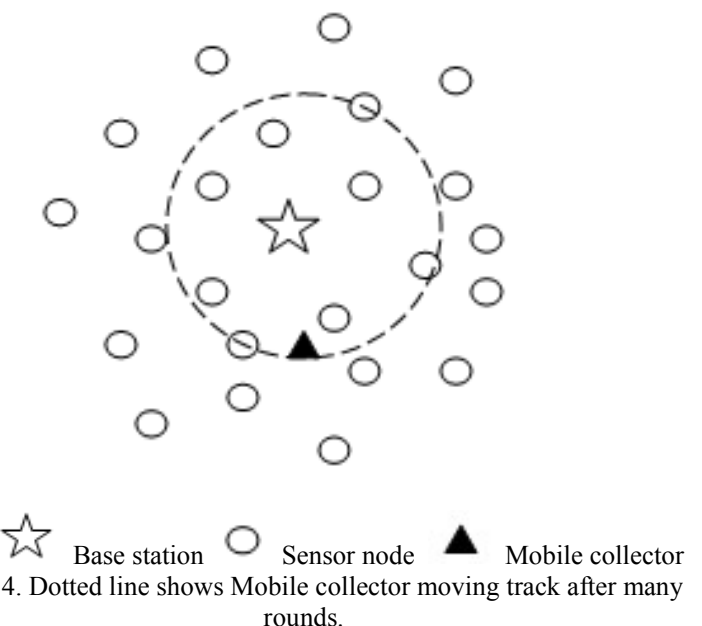

\section{Conclusion and Analysis}

Through the above analysis, we can see that the Non-uniform cluster-based Mobile data collector protocol is superior to traditional clustering protocol, better to alleviate hot zone problem near the base station, thereby extending the life of the network.

\section{ACKNOWLEDGMENT}

This paper is based upon work supported by Project by Scientific Research Fund of Hunan Provincial Education Department (No: 10B034), Hunan Provincial Natural Science Foundation of China ( No: 10JJ3067)
[1] G. Xing, T. Wang, Z. Xie, and W. Jia. Rendezvous planning in mobility-assisted wireless sensor networks. In IEEE RTSS, Dec 2007.

[2] Muhammad Arshad, Nasrullah Armi, Nidal Kamel, N.M. Saad, "Mobile data collector based routing protocol for wireless sensor networks," Scientific Research and Essays Vol. 6(29), pp. 6162-6175, 30 November, 2011, DOI: 10.5897/SRE11.1339 ISSN 1992-2248 (C)2011 Academic Journals

[3] R. C. Shah, S. Roy, S. Jain, and W. Brunette. Data mules: modeling a three-tier architecture for sparse sensor networks. In IEEE SNPA, pages 30-41, 2003.

[4] A. Somasundara, A. Ramamoorthy, and M. Srivastava. Mobile element scheduling for efficient data collection in wireless sensor networks with dynamic deadlines. In IEEE RTSS, pages 296-305, 2004.

[5] W. R. Heinzelman, A. Chandrakasan, H. Balakrishnan, "Energy-efficient communication protocol for wireless micro-sensor network," Proc. of the 33rd Intl Conf on System Science, pp.1-10, 2000 .

\section{REFERENCES}

